\title{
Development of Power Manipulator for remote handling in Nuclear Programme
}

\author{
Jagadishan D, Ravikumar R, Ulagathan , Kasiviswanathan K V, Sakthivel S \\ Group for Remote Handling, Irradiation Experiments, Robotics and PIE, \\ Indira Ganthi Centre for Atomic Research, Kalpakkam.603 102. \\ E-mail: jagan@igcar.gov.in, rrkr@igcar.gov.in
}

\begin{abstract}
The fabrication of advanced nuclear fuel material involves handling of highly radioactive material in high purity inert gas atmosphere. All the process operations and associated handling have to be necessarily carried out using remote in-cell techniques. These Radioactive cells essentially require handling equipments such as an in-cell crane and power manipulator for heavy handling operations that would be carried out remotely. Design of this equipment is usually done according to the requirement of remote operations which are needed to be carried out and suited to the layout of the radioactive cells or containment boxes. A power manipulator has been designed and developed for the use in IGCAR, Kalpakkam. The developmental efforts and performance in the power manipulator is discussed in this paper.
\end{abstract}

Key words: Radioactive, Nuclear fuel, Power Manipulator, in-cell manipulator.

\section{INTRODUCTION}

Power manipulator is used for handling heavy loads and performing the tasks which do not require force feed back. It is mounted on the crab of the special in-cell crane with common long travel to access full volume of the containment box. A power manipulator of $10 \mathrm{~kg}$ payload capacity has been designed and developed to operate in high purity argon atmosphere and it is modular and compact. Modularity of the system is essential for remote maintenance and assembly. Since the space inside of the process containment box is at premium, compact design and high work volume of Power Manipulator to containment volume ratio are the design criteria considered during the design stage. Careful selection of motors for the system has been of paramount importance because of the low ionization potential of the argon atmosphere in the containment box. The electrical equipments to be operated inside the containment box should be operable with power supply voltage not exceeding 110V, $50 \mathrm{~Hz}$ AC, since Breakdown Voltage of Argon gas is less.

Brushless DC (BLDC) servo motors have been selected for all motions to achieve compactness, maintenance-free operation, high mean time between failures (MTBF), high efficiency, and wide range of speeds. In addition, features like high torque to weight ratio and power output to frame size ratio makes BLDC motors ideal for such applications, where space and weight are critical.

\section{POWER MANIPULATOR}

The power manipulator has seven degrees of freedom viz, Cross travel (C.T), Azimuth (rotation about vertical axis), Vertical "Z" Motion, Shoulder Arm, Elbow Arm, Wrist Extension, Wrist Rotation, in addition to gripping motion of the tongs. The Power manipulator is operated through remotely operated handheld pendent and can be interfaced with PC for the computer controlled operations. The pendent is connected with the motion controller and variable frequency drives. Major material of construction of the power manipulator is Stainless Steel Type AISI 304 in view of the corrosive environment in the process containment box. Figure 1 shows the $3 \mathrm{D}$ model of the Power Manipulator,. Figure 2 shows the work volume which can be achieved due to the special joint design and the extent of angular motion which can be given to the various joints.

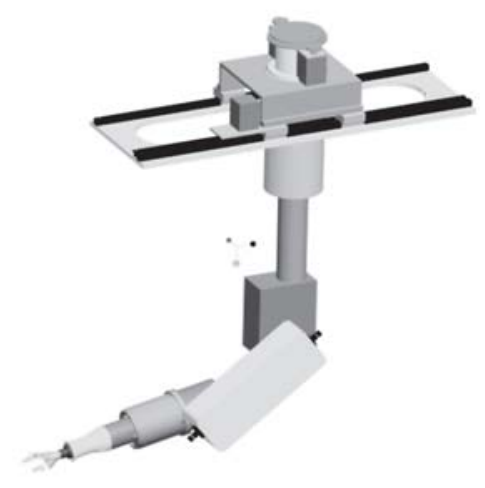

Fig 1. Power Manipulator of $10 \mathrm{Kg}$ Payload 


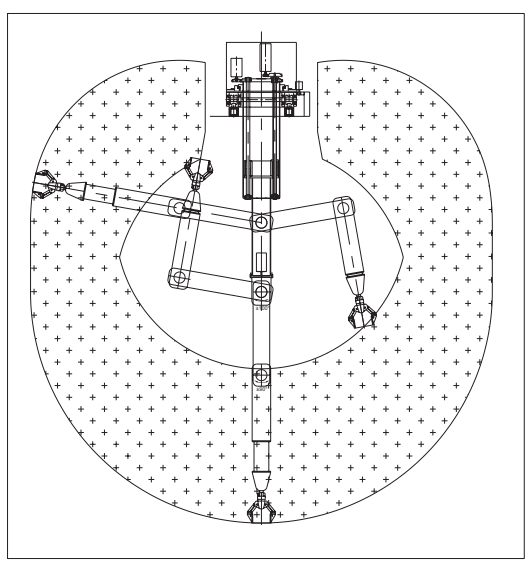

Fig 2 : Work Envelop of the Power Manipulator

\section{CONSTRUCTION OF POWER MANIPULATOR}

Figure 3 Shows A Photograph of The Power Manipulator

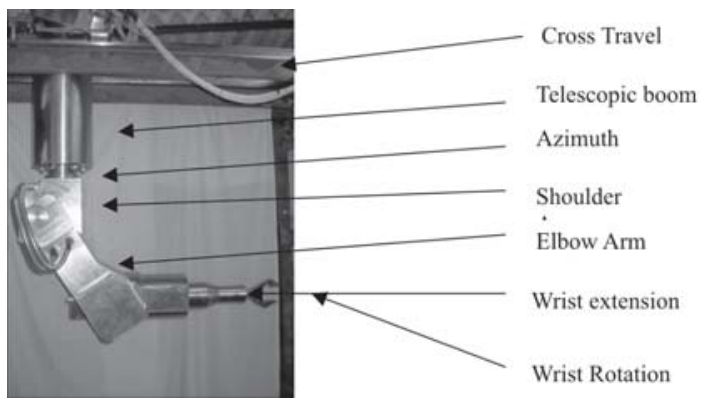

Fig 3 : Photograph of Power Manipulator

\section{CROSS TRAVEL}

The Power manipulator is fixed on the wheel carriage of the In-cell crane. A cross travel is provided with rack and pinion mechanism guiding on linear motion guide system. Cross Travel (CT) can travel with power manipulator. Cross travel of power manipulator and long travel of In-cell crane to enable to access over all length and width of the containmentbox.

\section{TELESCOPING BOOM MECHANISM:}

A single stage telescopic boom is fixed to extend the reach of the Power manipulator. The telescopic boom has been guided precisely with corrosion resistant guide rods. A mounting has been provided at the top of the telescopic boom to fix with the crane trolley. A junction box is provided on the end of the telescopic tube, from where the cables are distributed internally.

\section{AZIMUTH}

The rotating mechanism housed at the top of the fixed boom tube. The rotational range of the azimuth is 370 degrees about the vertical axis. The over lap of ten degree is provided to cover the entire circle and the restriction is provided to avoid the cable entangling.

\section{SHOULDER ARM SWIVEL}

Shoulder arm swivel is coupled to the bottom end of telescopic boom tube. It has a rotational range of \pm 100 degrees about the vertical axis. A compact AC brushless motor has been provided inside the shoulder. The motor is controlled through Control panel kept out side the cell.

\section{ELBOW SWIVEL}

Elbow axis is fixed at the shoulder axis module and both elbow and shoulder axes have rotational motions which enhance the dexterity of the manipulator.

\section{WRIST EXTENSION}

A wrist extension is provided in elbow swivel arm to facilitate increase the reach of gripper. The wrist extension has a linear travel of $150 \mathrm{~mm}$ and it is guided properly with corrosion resistant guide rods. A compact AC brushless motor is provided inside the wrist.

\section{WRIST ROTATION}

A wrist has been provided with endless rotation of gripper. It is fixed to the end of the elbow. A very compact AC brushless inbuilt brake motor is provided inside the wrist to have the endless rotation. The wrist will rotate about the elbow swivel arm axis. A inbuilt brake motor is used in the wrist rotation so that in case of power failure, the wrist should not allow the object to fall freely either because of gravity or due to shifted C.G. of the object or any other external force.

\section{GRIPPER}

A two finger parallel jaw gripper is provided at the end of wrist assembly. A suitable arrangement has been provided in the wrist and its interface to accommodate wrist rotation motor as well as gripper motor inside the wrist body itself. The gripper has the maximum opening of 100 $\mathrm{mm}$. Provision has been made to replace the finger remotely. The arrangement is made to provide necessary gripping force for handling $10 \mathrm{~kg}$ payload. A force sensor has been provided to control the gripping force. Users can able set the gripping force from the selector depending upon the requirement. Users can set the gripping force in the control panel depending upon the nature of the object being handled. Speed of each axis can be varied using the pendant according to the requirement. Figure 4 shows the photograph of the parallel jaw grippers used in the power manipulator. 


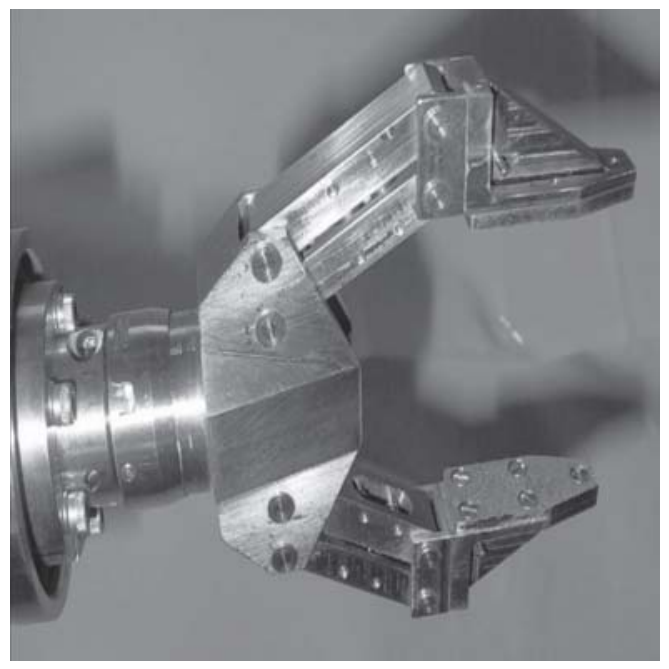

Fig 4 : Photograph of Parallel Jaw Grippers

\section{RESULTS \& DISCUSSION}

Table 1 gives the salient specification of the Power Manipulator with respect to the motion requirements and speed of the travel of each axis.

The manipulator has been tested for functionality at various speeds to evaluate the complete performance as given in the table-1. The force sensor for gripping force has been calibrated with standard load cell. In the fully extended condition the maximum overall deflection is 9.2 $\mathrm{mm}$ with maximum payload applied at the tip of the wrist, which well within the expected level.
Table 1 : Broad Specification of the PM of Payload Capacity $10 \mathrm{~kg}$

\begin{tabular}{|l|l|l|l|}
\hline $\begin{array}{r}\text { SI } \\
\text { No }\end{array}$ & \multicolumn{1}{|c|}{ Axis } & \multicolumn{1}{c|}{ Travel } & \multicolumn{1}{c|}{ Speed } \\
\hline 1. & Cross Travel (C.T) & $1500 \mathrm{~mm}$ & $0-25 \mathrm{~mm} / \mathrm{s}$ \\
\hline 2. & Z - Axis & $375 \mathrm{~mm}$ & $0-10 \mathrm{~mm} / \mathrm{s}$ \\
\hline 3. & Azimuth & $370^{\circ}$ & $0-9 \mathrm{deg} / \mathrm{s}$ \\
\hline 4. & Shoulder & $+/-100^{\circ}$ & $0-8 \mathrm{deg} / \mathrm{s}$ \\
\hline 5. & Elbow & $+/-90^{\circ}$ & $0-10 \mathrm{deg} / \mathrm{s}$ \\
\hline 6. & Wrist extension & $150 \mathrm{~mm}$ & $0-8 \mathrm{~mm} / \mathrm{s}$ \\
\hline 7. & Wrist rotation & Endless & $0-20 \mathrm{deg} / \mathrm{s}$ \\
\hline 8. & Gripper & $100 \mathrm{~mm}$ & $0-10 \mathrm{~mm} / \mathrm{s}$ \\
\hline Over all dimensions of the Power Manipulator \\
\hline \multicolumn{4}{|l|}{ Fully collapsed condition } \\
\hline \multicolumn{4}{|l|}{ Fully extended condition } \\
\hline
\end{tabular}

The special feature of this manipulator is modularity in design to reduce downtime during maintenance. Positional read out for all axes are incorporated to know position of the each arm.

\section{CONCLUSION}

Manipulators and Robots are needed most in areas where human finds it dangerous or impossible to work. In nuclear installations, radioactivity poses problem in access, work, repair and maintenance. This Power manipulator is developed in open loop, but it can be automated by providing feed back system.

\section{ACKNOWLEDGMENT}

The authors are thankful to Dr. S. Venugopal, Head, RIRD, IGCAR for his keen interest and valuable suggestions and guidance. The authors also thankfully acknowledge the contributions of many of their colleagues in RIRD, GRIP, IGCAR, Kalpakkam. 